\title{
Modernizar la administración a través de la participación: la experiencia de la Generalitat de Catalunya
}

\author{
Quim Brugué Torruella \\ Universidad Autónoma de Barcelona \\ quim.brugue@uab.cat
}

\section{Resumen}

La experiencia de la Generalitat de Catalunya muestra la bondad de la participación ciudadana a nivel autonómico. El modelo de participación en la distancia puede aprender de las experiencias locales, pero, al mismo tiempo, debe ser capaz de replantear algunas de las ideas dominantes en este ámbito, de forma que el debate entre vinculación y consulta resulta irrelevante. Es necesario diseñar un modelo basado en estrategias transversales que den lugar a deliberaciones y procesos participativos, para garantizar una responsabilidad pública capaz de hacer frente a los compromisos politicos y a las necesidades de la colectividad.

Palabras clave: administración, participación, democracia deliberativa.

\begin{abstract}
The good experience of the Catalan government (Generalitat) is an example of citizen participation at an autonomous level. The model of distant participation can learn from local experiences, but should also discuss some of the field's dominant issues, so that the debate among entailment and consulting becomes irrelevant. A new model design based on transversal strategies can give way to deliberations and participative processes. Such processes must guarantee public responsibility in order to face political commitments for the collective demands.
\end{abstract}

Key-words: public administration, participation, deliberatie democracy. 


\section{Dirección General de Participación Ciudadana ${ }^{1}$}

Durante los últimos años, sobre todo en el ámbito local, han proliferado los debates y las experiencias de participación ciudadana. No queremos hacer un recorrido académico por definiciones, aproximaciones y tipologías, pero sí destacar un par de ideas que se han ido consolidando y que quizás deberemos replantear, especialmente cuando las apliquemos en el ámbito autonómico.

En primer lugar, se suele justificar la participación en función de la proximidad o, con otras palabras, del conocimiento de la realidad sobre la que se quiere actuar. Es este conocimiento el que justifica la necesidad de escuchar y de tomar en consideración las opiniones de los ciudadanos-conocedores-próximos.

En segundo lugar, y como consecuencia del punto anterior, es habitual evaluar la participación en función del mayor o menor compromiso con aquello que expresan los ciudadanos-conocedores-próximos. Así, se considera "participación de baja intensidad» la que consulta a la ciudadanía, mientras que se califica de "participación de calidad» la vinculante, es decir, la que le cede la capacidad efectiva para tomar las decisiones.

La primera idea sitúa al ámbito autonómico en una posición complicada, puesto que no dispone del factor proximidad y, por lo tanto, de los ciudadanos-conocedorespróximos que justifican la bondad de la participación. Por tanto, hay que pensar cómo se justifica la participación autonómica: una participación desde la distancia. Por otro lado, sin esta proximidad tampoco queda muy claro el debate entre participación consultiva o vinculante. No es lo mismo discutir cómo se incorpora la opinión de un número reducido y más o menos asequible de ciudadanos-conocedores-próximos que plantearlo para más de siete millones de personas, dispersas por el territorio y con conocimientos limitados sobre las políticas autonómicas. Los interrogantes que se plantean no son, pues, menores: ¿puede el tamaño autonómico asumir fórmulas de democracia directa (consultiva o vinculante) sin entrar en metodologías demoscópicas que garanticen la representatividad de estos más de siete millones de personas? Y, si es así, ¿̇es aquí donde se encuentra la innovación democrática, en una democracia por sondeo?

Probablemente se ha tendido a responder a estos interrogantes, sobre los que volveremos más adelante, con un no, no y no; es decir, con poco interés por la innovación democrática en el ámbito autonómico: no hace falta porque la distancia no lo recomienda, no hace falta porque no acabamos de ver cómo funciona la democracia directa a esta escala y, finalmente, no hace falta si de lo que se trata es de hacer una encuesta tras otra. No hace falta y, por lo tanto, como veremos en el próximo apartado, lo que

1 Ll. Alonso, C. Blesa, Q. Brugué, F. Català, J. M. Espuelas, C. Montoro, J. Pacheco y F. Pindado. 
hasta hace poco se había hecho sobre participación ciudadana en la Generalitat era claramente deficiente y limitado. Lo más difícil es pensar en una alternativa, en cómo construir una estrategia para fomentar la participación y el debate en el ámbito autonómico. Esta cuestión la abordaremos en la segunda parte del artículo.

\section{Una nueva estrategia para fomentar el diálogo y la participación ciudadana de la Generalitat de Catalunya}

Con la llegada del nuevo Gobierno, de acuerdo con el Pacto del Tinell, se manifiesta la voluntad de construir una Administración relacional, propia de las democracias avanzadas, donde las decisiones se tomen con la implicación de la ciudadanía. El Plan de Gobierno 2004-2007, en este sentido, determina que se deben definir procesos que permitan una relación más próxima entre los gestores públicos y la sociedad para mejorar el diseño y los resultados de las políticas públicas en una sociedad cada vez más compleja y diversa.

El Plan define diversos objetivos en materia de participación ciudadana:

1) Crear mecanismos de participación.

2) Definir el marco participativo para promover instrumentos y protocolos de actuación para escuchar a la ciudadanía.

3) Dar apoyo y asesoramiento a las políticas de participación ciudadana en el ámbito local.

Además, en respuesta a las deficiencias del modelo tradicional, se considera necesario impulsar unos órganos y unos procesos de participación ciudadana basados en los siguientes principios:

1) Coordinación. Buscar la coordinación de los departamentos para evitar que los procesos de participación interfieran unos con otros.

2) Integración. Combinar las políticas públicas y no favorecer actuaciones aisladas. La participación forma parte de procedimientos de toma de decisiones en unas políticas de carácter integral.

3) Descentralización. Acercar la Administración y el Gobierno a las personas y a los territorios.

4) Confianza. Buscar la deliberación franca, como base de la participación que busca el consenso, en la que se precisa de confianza entre los participantes.

5) Apertura. Mejorar la representatividad de las personas participantes y permitir, cuando sea razonable, la presencia de personas a título individual. 
6) Actitud propositiva. Redefinir los órganos y los procedimientos de participación e introducir, si es preciso, cambios normativos.

Inmediatamente, tras la constitución del Gobierno tripartito, se creó la Dirección General de Participación Ciudadana. Esta es la instancia que debe permitir pasar de las voluntades políticas a la práctica, a través de la definición y la puesta en marcha de una estrategia para el fomento del debate social y de la participación en las políticas autonómicas. Explicaremos los principales ejes de esta estrategia a partir de tres interrogantes muy elementales: ¿por qué lo hacemos, qué queremos hacer, y cómo proponemos hacerlo?

\section{¿Por qué lo hacemos?}

Este primer interrogante ya ha sido contestado en parte: porque nos lo creemos, porque estamos convencidos de que sin incorporar la ciudadanía a la participación y al debate no seremos capaces de dar respuesta a aquello que la sociedad nos está demandando. Dejadnos ir algo más lejos, porque pensamos que hoy, sin participación, no se puede gobernar. No podemos hacer una política educativa sin incorporar a maestros, alumnos, padres, etc. No podemos incidir sobre las deslocalizaciones de empresas sin contar con empresarios, sindicatos, universidades, etc. No podemos gestionar la inmigración sin la implicación del tercer sector, de las asociaciones, de los ayuntamientos, etc. No podemos trabajar por el respeto al medio ambiente sin las escuelas, sin las entidades ecologistas, sin los usuarios del medio natural, etc.

Pero, ¿por qué no podemos? Desde nuestra perspectiva, la implicación de todos estos actores es estrictamente necesaria desde una doble vertiente: la sustantiva y la operativa.

Sustantivamente, incorporar la ciudadanía enriquece las decisiones y favorece la innovación. Dado el grado de complejidad y de incertidumbre de las decisiones que hoy nos corresponde tomar, esta es una aportación capital. Dicho de otra manera, ante los poliédricos problemas actuales (hacer una carretera es un asunto de ingeniería, de economía, de ecología y de cohesión social, por poner un ejemplo), necesitamos respuestas poliédricas, respuestas que no se encuentran en un único lugar (la Administración), sino en varios (los diversos actores sociales).

Operativamente, pese a las percepciones dominantes, la participación sirve para mejorar la eficiencia y la eficacia de la actividad gubernamental y administrativa. Incorporar los diferentes actores sociales puede generar costes adicionales al inicio del proceso, pero estos se recuperan — con creces — en la medida en que la participación sirve 
para reducir resistencias y conseguir colaboraciones. Pensemos, por ejemplo, en las dificultades de construir prisiones, vertederos o centros para drogodependientes desde la soledad del despacho de quien tiene la responsabilidad de construirlos. No estamos insinuando que se deba renunciar a esta responsabilidad; afirmamos, por el contrario, que esta responsabilidad se puede ejercer con más eficiencia y eficacia.

Se intuye que nos dirigimos hacia un modelo de participación donde el debate entre consulta y vinculación no es demasiado relevante. La participación vinculante - la de calidad para muchos observadores y analistas - no tiene demasiado sentido cuando hemos de escuchar muy diversas voces y atender a intereses muy diferentes. ¿Cuál es la opinión vinculante, la de quien piensa $a$ o la de quien piensa $b$ ? Evidentemente, si hay quien piensa $a$ y quien piensa $b$, podemos caer fácilmente en la tentación de dejarlo para más adelante, para cuando se pongan de acuerdo. Si no podemos consensuar dónde situar un centro penitenciario, ¿qué hacemos? ¿Dejamos de construirlo? ¿Hacemos una encuesta? Obsesionarnos con el carácter vinculante de la participación nos puede conducir a la parálisis y, por tanto, al fracaso de aquello que hemos fijado como objetivo básico de la participación: contribuir a resolver los problemas de la ciudadanía... y las prisiones lo continuarán siendo, aunque no decidamos dónde situarlas.

Así pues, la conclusión es que, al menos en el ámbito autonómico, la democracia directa vinculante nos conduce a un callejón sin salida. La alternativa demoscópica no tiene sentido (hacer una encuesta imposible para decidir dónde construir la prisión, si gana a o gana $b$ ), de manera que debemos pensar en alguna otra estrategia. Para nosotros, esta alternativa es la democracia deliberativa. Lo que debemos hacer es organizar procesos o espacios deliberativos, pero esto nos plantea el segundo interrogante.

\section{¿Qué debemos hacer?}

Siguiendo con el razonamiento, tenemos que organizar deliberaciones, diálogos, procesos de debate a través de los cuales conseguir los dos objetivos fijados anteriormente: enriquecer las decisiones y mejorar la eficacia de las actuaciones. Es con estos diálogos con y entre los diferentes actores institucionales, sociales, económicos, etc. como conseguiremos las visiones poliédricas (decisiones más ricas) y las complicidades necesarias (actuaciones más eficaces).

Pero ¿qué quiere decir organizar una deliberación o un diálogo? Desde nuestro punto de vista, significa estructurar un proceso o un espacio en el que garantizamos que varios actores dicen cosas, se escuchan unos a otros y sacan conclusiones útiles y operativas. Esto, que parece tan elemental, es de una gran complejidad, sobre todo porque a menudo todos nosotros hablamos sin saber muy bien qué decimos, porque 
casi nunca escuchamos a nadie y porque nuestras conversaciones acostumbran a acabar sin acuerdos ni conclusiones.

¿Qué debemos hacer? Fundamentalmente, trabajar para superar estas dificultades. Las metodologías para conseguirlo pueden ser muy diversas - ahora no vamos a entrar en detalle-, pero se deben desplegar teniendo presente que la estructura de la deliberación, del diálogo, siempre debe satisfacer las fases siguientesł explicarnos, escuchar, tomar nota y pasar cuentas.

a) Explicarnos: el primer momento de la participación es la comunicación, y esta debe incorporar dos ingredientes básicos, que son la pedagogía y la honestidad. Cuando se invita a alguien a deliberar sobre un determinado asunto, de entra$\mathrm{da}$, es preciso ofrecerle toda la información y las explicaciones necesarias. Solo así podrá hablar con conocimiento de causa y decir cosas interesantes y pertinentes. También tenemos que ser honestos y explicar con mucha claridad de qué queremos hablar y de qué no, y cuáles son los límites de lo que estaríamos dispuestos a incorporar en nuestras políticas. Para expresarlo con un ejemplo, no podemos comenzar un proceso participativo sobre el Estatuto sin explicar qué es y de qué trata. Además, al comenzar este proceso, debemos ser honestos y decir a quien opine que es preciso reducir nuestro nivel de autogobierno o que hemos de integrarnos en el Estado francés; que serán escuchados, pero no tomados en consideración. La innovación democrática puede completar la democracia representativa, no traicionarla.

b) Escuchar: esta fase incorpora el despliegue de canales e instrumentos para recibir de manera ordenada las aportaciones de la ciudadanía. Podemos pensar en infinidad de instrumentos (jornadas, páginas web, talleres, etc.), pero lo único que ahora queremos subrayar es la importancia de desplegar una diversidad de canales suficiente para permitir que los diferentes participantes encuentren vías de entrada. En un debate no es tan importante el número de participantes como que todo el mundo que quiera participar pueda hacerlo, que todo el mundo encuentre un espacio - esto no siempre es fácil— donde se sienta cómodo para hablar. Unos lo harán a través del ordenador, otros en una reunión y unos cuantos jugando en la plaza.

c) Tomar nota: lo que dice la gente no nos puede entrar por una oreja y salir por la otra. Hace falta que lo analicemos y lo sistematicemos, de forma que pueda ser (o no) incorporado al proceso decisorio. Lo que más molesta a la gente no es que su aportación no sea incorporada, sino que sea ignorada: aceptamos un no, pero nos ofende el silencio.

d) Pasar cuentas: como final lógico de la fase anterior, hemos de acabar pasando cuentas de lo que hemos incorporado y de lo que hemos desestimado, y expli- 
car el porqué. La ciudadanía se ha tomado la molestia y ha asumido el coste de ir a reuniones o de conectarse a Internet. Es por mera simetría que ahora corresponde a los receptores de estas opiniones tomarse la molestia de responder a los participantes en el debate. De nuevo, esta es una fase compleja, sobre todo si no se ha planificado con anterioridad.

\section{¿Cómo debemos hacerlo?}

El cómo es siempre la parte más difícil de resumir y, en cualquier caso, se puede valorar mejor a través de las experiencias que de los discursos. No obstante, queremos destacar dos reflexiones de carácter genérico en relación con el cómo: la primera es estratégica y tiene que ver con cómo impulsar la participación desde una dirección general hacia los restantes departamentos (el trabajo transversal); la segunda es metodológica y tiene que ver con los elementos básicos para entender cómo se ponen en marcha procesos y espacios de participación y deliberación. Nos referiremos al segundo "cómo", esto es, a los procesos y espacios de participación, en el próximo apartado; ahora nos centraremos en el primero.

Cualquier persona con un mínimo de experiencia en la Administración sabe cuál es el final de la gran mayoría de los proyectos que reclaman trabajo transversal: el fracaso. Pues bien, la Dirección General de Participación Ciudadana debe trabajar transversalmente, aun cuando no está destinada inexorablemente al fracaso. Ahora bien, para no fracasar, lo primero que se necesita es una estrategia de relación con los otros departamentos basada tanto en la identificación de las dificultades de la transversalidad como en una acción explícita para contrarrestarlas.

¿Cuáles son los principales problemas para este trabajo transversal en el que la Dirección General de Participación Ciudadana quiere fomentar y ayudar a organizar procesos y espacios de debate en otros departamentos y sobre temas que le son ajenos? Podríamos utilizar el recurso de la triple-D para responder esta pregunta: desconocimiento, desconfianza y día a día.

a) Desconocimiento. Hablar hoy de participación es un lugar común, de manera que son muchos los departamentos y las unidades administrativas que utilizan este concepto a la hora de pensar en sus planificaciones y actividades. Esto debería ayudar al trabajo transversal, puesto que produce un lenguaje y unos planteamientos comunes sobre los que trabajar juntos. El problema radica en la gran confusión que hay sobre el concepto y la operativa de la participación. Así, es frecuente que las visiones no coincidan, que no todo el mundo sepa exactamente de qué está hablando y que exista un gran desconcierto sobre cómo 
ejecutarla. En este escenario, la transversalidad resulta difícil, puesto que echa de menos la comprensión compartida sobre la que trabajar juntos.

b) Desconfianza. Incluso cuando hay cierta coincidencia en los planteamientos participativos, estos se deben desarrollar en ámbitos sustantivos, como el bienestar, la sanidad, la ordenación territorial o los planes energéticos. En estos ámbitos, los departamentos tienden a monopolizar el conocimiento experto $y$, por tanto, desconfían de la incursión de personas ajenas. Dicho más claramente, dejar que alguien de fuera organice un debate $y$, por tanto, se ponga a hablar con actores sociales sobre energía, ordenación territorial o educación despierta desconfianza. ¿No dirán algo inoportuno? ¿Adquirirán compromisos que no podremos cumplir? ¿Despertarán expectativas que no podremos controlar? ¿Asumirán un protagonismo que no les corresponde?

c) Día a día. La participación - lo comentábamos antes - debe servir para fomentar la eficiencia y la eficacia de las actuaciones, aun cuando en sus inicios puede comportar costes en términos de tiempo, recursos y esfuerzos. Estos costes iniciales a menudo son difíciles de asumir, puesto que se muestran incompatibles con la presión del día a día. Pese a las buenas voluntades, suele ocurrir que la acumulación de tareas cotidianas y la presión por hacer, hacer y hacer no deja espacio para incorporar unos procesos que, de todos modos, no existían hasta ahora y que nadie reclama con la misma intensidad que otros asuntos más ligados a la inmediatez.

¿Cómo se debe responder a las dificultades que plantea la triple-D? Es obvio que no existe ninguna varita mágica, pero pensamos que, frente al desconocimiento, la desconfianza y el día a día, hace falta ofrecer mucha comunicación, mucha confianza y mucha generosidad:

a) Comunicación. Hace falta, en primer lugar, desplegar un plan de comunicación interno que sirva para explicar cuál es el modelo de participación, qué ventajas generará y cómo se llevará a cabo. Este plan de comunicación requiere, sobre todo, un fuerte liderazgo político que sirva tanto para asegurar la seriedad de la apuesta como para favorecer el acuerdo y el compromiso bajo la base común necesaria para el trabajo transversal.

b) Confianza. La confianza no se impone, sino que se debe ir ganando. Esto, que es tan elemental, exige una estrategia que requiere de cierta paciencia, conscientes de que hace falta ir mostrando a los otros departamentos que, cuando nos abren la puerta, no queremos complicarles la vida, sino ayudarles; que no les quitaremos protagonismo, sino que se lo cederemos; que no diremos nada inoportuno, porque ellos determinarán los contenidos. Aparte de demostrarlo 
con la experiencia, esta confianza debe construirse a partir de un equipo técnicamente muy solvente y profesionalmente muy fiable.

c) Generosidad. La participación genera costes y beneficios y, a menudo, los beneficios no llegan por la dificultad inicial de asumir los costes. La tarea de una dirección general de participación debería ser asumir los costes, dejando que los beneficios se queden en los departamentos. Si este es el planteamiento, conseguiremos que la presión del día a día no bloquee estas experiencias, puesto que la brecha no la deberá asumir el mismo departamento. «Para mí, los costes; para ti, los beneficios»: en la generosidad radica el truco para hacer efectiva la transversalidad.

Hasta aquí hemos dibujado las grandes líneas en las que está trabajando la Dirección General de Participación Ciudadana de la Generalitat de Catalunya. Se trata de unas líneas que empiezan a trazarse desde la convicción de la utilidad de la participación para mejorar la capacidad de respuesta de los poderes públicos a las demandas de la ciudadanía, y que ganan peso a través del concepto de "democracia deliberativa» y de una estrategia para la transversalidad. Pensamos, por tanto, que sí: que sí es preciso diseñar un modelo de participación desde la distancia. Pensamos también que este modelo puede aprender de las experiencias locales, pero que, al mismo tiempo, debe ser capaz de replantear algunas de las ideas dominantes que se han instalado en este ámbito. En este sentido, apostamos por generar diálogos y deliberaciones que enriquezcan decisiones y mejoren actuaciones. Lograrlo comporta muchas dificultades, pero si afrontamos el reto con seriedad obtendremos excelentes resultados. No tenemos ninguna duda.

\section{Procesos de debate y deliberación}

Cuando hablamos de impulsar procesos de debate y deliberación social nos referimos a procesos que se desarrollan puntualmente y que tienen por objetivo redactar una ley, aprobar un plan o programa, diseñar una política o iniciar una planificación estratégica. Es decir, nos referimos a cómo implicamos a la ciudadanía en debates o deliberaciones sobre la definición de políticas, las cuales, una vez llevadas a cabo, deben ser administradas por las diferentes instancias responsables. En definitiva, estamos incorporando a los ciudadanos y ciudadanas en el proceso decisorio, en aquello que denominamos «la política».

Nos hallamos, pues, ante una propuesta importante y que nos obliga a ser capaces de combinar y hacer compatibles dos aspectos que no siempre se acomodan con facilidad: 
1. Las deliberaciones y los procesos participativos que sirven para incorporar opiniones que, como hemos subrayado en el apartado anterior, deben mejorar las decisiones y las actuaciones.

2. La responsabilidad pública en el momento de hacer frente a los compromisos políticos y a las necesidades de la colectividad. El trabajo político no consiste en identificar y responder al interés (único) de la ciudadanía, sino en observar los intereses existentes (varios) y en buscar una respuesta que, o bien los equilibre, o bien defienda aquellos que explícitamente se apuesta por primar. Hacer política con responsabilidad, por tanto, no es hacer lo que pide la gente (no existe tal cosa), sino tomar con claridad y transparencia las decisiones que se consideran adecuadas para la colectividad. Esta responsabilidad no se puede dejar de ejercer ni puede quedar disimulada por un proceso participativo.

A menudo tanto los excesivamente críticos como los excesivamente entusiastas con la participación olvidan la necesidad y la posibilidad de combinar ambos elementos. Los críticos dirían que apostar por la participación es una manera de debilitar la responsabilidad pública, de forma que, al final, ni se toman decisiones ni se da cuenta de ellas. Para los entusiastas, por el contrario, a veces parece que la participación solo lo es de verdad si sustituye a unos poderes públicos que se deben limitar a escuchar y a hacer caso a la ciudadanía. Nosotros pensamos, en cambio, que el éxito reside en la combinación de ambas posiciones; una combinación que, de hecho, nos saca del debate entre democracia representativa "o» participativa y nos sitúa en el terreno de la democracia representativa "y» participativa. Es preciso escuchar a la ciudadanía e incorporar sus opiniones, pero esto no significa renunciar ni a los compromisos políticos ni a las responsabilidades públicas. En términos operativos, la clave reside en cómo conseguir hacer compatibles estas posiciones. Se trata, en fin, de evitar los extremos.

Encontrar el punto de equilibrio siempre es difícil y, en este sentido, el trabajo de la Dirección General de Participación Ciudadana es ofrecer metodologías, protocolos y asesoramiento para conseguirlo. Como siempre, desgraciadamente, no hay recetas mágicas, aunque sí nos atrevemos a proponer un guión de trabajo. Este se basa en una secuencia de cinco fases pensadas para hacer compatible la responsabilidad pública y la participación ciudadana. Estas fases son:

1. Definir el marco de referencia

2. Difundir el proyecto

3. Organizar y ordenar espacios de participación y debate

4. Redactar las conclusiones del proceso

5. Ejercer la responsabilidad pública 


\section{Definir el marco de referencia}

Antes de iniciar cualquier proyecto de debate social en torno a un programa, un plan o una pieza legislativa, es preciso disponer de un marco de referencia. Este es un primer ejercicio de responsabilidad pública. Pero, ¿̇en qué consiste un marco de referencia? Este debe representar la honestidad que reclamábamos en el artículo anterior; es decir, consiste en hacer explícitos los ejes básicos del proyecto público que se quiere impulsar, los límites del debate que se quiere abrir, las líneas políticas de partida, los aspectos sobre los que se está dispuesto a hablar y los que representan una opción política que no se quiere dejar de asumir.

Este marco de referencia se debe poder explicar con la máxima claridad a la sociedad y, por esto, proponemos hacerlo a través de un guión de preguntas-respuestas. Estas, además, se pueden agrupar en dos grandes paquetes: las que sirven para explicar los contenidos del proyecto $y$, por tanto, para conseguir participantes informados; $y$ las que sirven para fijar los límites y, por tanto, explicitar el espacio de responsabilidad pública que se quiere preservar.

En cuanto al primer bloque, sugerimos un argumentario que aborde de manera sencilla algunas preguntas elementales, como las siguientes:

a) ¿Cuál es el plan, el programa o el proyecto que nos ocupa?

b) ¿Para qué sirve y qué queremos conseguir con él?

c) ¿De qué habla? ¿Cuáles son los temas que trata?

d) ¿Cómo se quiere debatir el proyecto?

Si lo que queremos es abrir un debate para redactar una nueva Ley de Servicios Sociales, por ejemplo, debemos explicar a los participantes potenciales qué es la Ley de Servicios Sociales, de qué temas habla o puede hablar, cuáles son los objetivos que se persiguen, por qué se quiere hacer esta ley de nuevo o por qué se quiere modificar la que ya existe, cómo será el proceso de elaboración, etc. Si no sabemos dar estas explicaciones, difícilmente podremos abrir un debate mínimamente pertinente.

Respecto al segundo bloque, el mensaje que demos debe dejar claros algunos límites, como los siguientes:

a) ¿Sobre qué temas queremos discutir y sobre cuáles no?

b) ¿Cuáles son los criterios o las categorías para la discusión?

c) ¿Cuáles son los puntos conflictivos?

d) ¿Cuáles son las posiciones «fuertes» de los responsables públicos? 
e) ¿Hay condicionantes técnicos, económicos, jurídicos o de otra naturaleza que haya que tomar en consideración?

f) ¿Cuándo se acabará el proceso de participación?

g) ¿Qué condiciones o reglas establecemos para el debate?

Volviendo al ejemplo de la Ley de Servicios Sociales, el marco de referencia debe establecer los temas de discusión; en este sentido, por citar un ejemplo real, el documento de bases elaborado y difundido desde el Departamento de Bienestar y Familia es una buena muestra de cómo fijar el guión para un debate ordenado y pertinente. Asimismo, habría que ser claros en relación con los temas en que las posiciones políticas estén tomadas o en relación con los condicionantes que se deben tomar en consideración. Finalmente, los debates no pueden ser eternos ni caóticos, de manera que es preciso definir las reglas y los plazos.

\section{Difundir el proyecto}

Una vez definido el marco de referencia, se abre una fase de difusión del plan que debe servir para hacer llegar el argumentario al conjunto de la sociedad civil. Así, se trata tanto de situar el tema en la agenda pública como de explicar a la población los contenidos básicos y la importancia del tema que se trata. Solo de esta manera el debate puede resurgir y hacerse con interlocutores interesados e informados.

Esta fase, dependiente de los recursos disponibles en cada caso, requiere de acciones vinculadas a:

a) el diseño de una estrategia integral de comunicación: mensajes centrales, artículos, entrevistas, etc;

b) la producción de materiales destinados a la difusión, tanto a través de medios de comunicación como de otras vías: trípticos, anuncios, logotipos, webs, etc.

También es importante identificar los diferentes colectivos a los que queremos hacer llegar nuestras explicaciones y adaptar, en función de sus características, los materiales y las acciones divulgativas. En este sentido, por ejemplo, en la campaña del Estatuto se elaboraron anuncios para los medios de comunicación (televisión y prensa) para dirigirse al conjunto de la población, material escolar para los jóvenes, folletos explicativos para las entidades de la sociedad civil, banners para las personas usuarias de las redes electrónicas, y trípticos explicativos para repartir en los actos que se hacían por todo el territorio. 


\section{Organizar y ordenar espacios de participación y debate}

Paralelamente a la fase de difusión, se debe desarrollar la fase más específicamente participativa. Esta, en función de cada caso, puede contar con un amplio abanico de iniciativas, pero siempre debería tener en cuenta los siguientes puntos:

a) Debe dirigirse a las entidades de la sociedad civil organizada y a las instituciones: esto quiere decir identificarlas, pedir su opinión (bilateralmente) y convocarlas a debatir — organizadamente - sobre los aspectos más relevantes del tema en cuestión (sesiones multilaterales).

b) Debe dirigirse a las personas a título individual, combinando la explicación pedagógica con la apertura de espacios donde puedan hacer llegar sus reacciones y opiniones.

c) Debe tener en cuenta la dimensión territorial y, por tanto, planificar estrategias y actuaciones para acercarse a la ciudadanía a través de los ayuntamientos, de las universidades, de las delegaciones del Gobierno, de sus entidades, etc.

En todos los casos, el proceso debe ser muy claro en relación con las metodologías, los calendarios, la necesidad de recibir las aportaciones por escrito, etc. Por ejemplo, la nueva Ley de Juventud que se quiere redactar desde la Secretaría de Juventud de la Generalitat ha generado un proceso de debate social en el que, por una parte, se ha abierto un buzón electrónico para recibir las aportaciones individuales de los jóvenes, $y$, por otra, se han organizado encuentros territoriales y sectoriales para discutir un documento de bases con las principales organizaciones e instituciones que trabajan en el mundo de la juventud. El análisis de los mensajes recibidos, junto con las conclusiones de los talleres territoriales y sectoriales, deben permitir recoger las aportaciones poliédricas que pueden enriquecer la futura ley.

Adicionalmente, desde un punto de vista pragmático, en la organización de estos espacios o canales de debate y participación hay que preocuparse, como mínimo, de un par de aspectos:

a) Difundir suficientemente los espacios e instrumentos que se ponen a disposición de la ciudadanía. No es menor, por tanto, el trabajo que hay que llevar a cabo para disponer de buenas listas de contactos, para hacer llegar las convocatorias a todo el mundo, para difundir los actos que se organicen, o para dar a conocer las posibilidades de participación que se están abriendo.

b) Diversificar los espacios y los canales de debate y participación de forma que se acomoden a los también variados gustos y sensibilidades de la población. Se 
trata de que todo el mundo que quiera participar encuentre su lugar, sin verse forzado a hacer acciones - conectarse a Internet, perder un sábado en una jornada, tener que rellenar un cuestionario- que, quizás, le incomoden.

Como ejemplo de la preocupación por llegar a todo el mundo, el Gobierno de la Generalitat ha impulsado la creación de un grupo interdepartamental que tiene como objetivo mejorar y actualizar el registro de asociaciones. Uno de los objetivos de esta iniciativa tiene que ver con la necesidad de disponer de datos fiables y exhaustivos para dirigirse a la sociedad civil.

Respecto a la importancia de la diversificación, el Estatuto nos ofrece un buen ejemplo, no exento de controversia. Aparte de la página web, las jornadas, los cuestionarios postales o los talleres territoriales, se representó el Festatut, una actividad de teatro participativo destinada a explicar el Estatuto en un lenguaje y un formato radicalmente diferentes. Muchas personas se han mostrado críticas con esta iniciativa, porque la han visto como una banalización de un asunto muy serio, del que solo se podía hablar en espacios igualmente serios. No entraremos en este debate, pero sí queremos subrayar un hecho: mucha gente no se sentía cómoda en el Festatut, pero, al mismo tiempo, determinadas personas que no se sentían cómodas ni en reuniones ni en ordenadores, sí que se sentían cómodas en esta actividad. Más concretamente, identificamos que la iniciativa tenía éxito cuando se situaba en barrios de población inmigrante (de otras partes de España o de otros países) o con presencia elevada de colectivos específicos, como, por ejemplo, personas del pueblo gitano. La iniciativa pudo tener más o menos éxito, pero fue el único espacio en el que pudimos llegar a estas personas.

\section{Redactar las conclusiones del proceso}

Una vez cerrada la fase participativa, es muy importante redactar un primer informe en el que se explique el proceso realizado y se recojan las aportaciones recibidas. Este informe debe hacerse público, se debe difundir y debe permitir un periodo de comentarios.

Esta fase puede parecer simple, pero comporta una dificultad operativa: el análisis y la síntesis de unas aportaciones que pueden ser muchas y muy diversas. Se trata de un trabajo que, además, reclama tanto un buen conocimiento del ámbito como una metodología de trabajo eminentemente artesanal, puesto que es muy difícil mecanizarlo o externalizarlo. Desde la Dirección General de Participación analizamos y sistematizamos las aportaciones recibidas en el debate sobre el Estatuto; otro tanto hemos hecho 
para el Departamento de Bienestar y Familia en relación con la futura Ley de Servicios Sociales.

En cualquier caso, los beneficios de hacerlo son muy relevantes, puesto que, si se difunde lo suficiente, este informe demuestra a los participantes que no solo se les ha dejado hablar, sino que además se les ha escuchado. En el apartado anterior ya hemos aludido a la importancia de este hecho.

\section{Ejercer la responsabilidad pública}

Finalmente, los responsables públicos deben valorar las aportaciones, decidir cuáles incorporan y cuáles no, y justificar sus decisiones. Pensamos que esta fase también se debe documentar y publicitar a través de un informe, puesto que es la manera de hacer algo que la teoría considera esencial: el retorno del proceso participativo, la explicación a los participantes de qué ha pasado con sus opiniones y aportaciones. Los participantes pueden aceptar que sus opiniones no sean incorporadas, si se les explica. Lo que no aceptan, en cambio, es que no se les ofrezcan estas explicaciones, que parezca que se ha celebrado un rito participativo, pero que, en realidad, ni se les ha escuchado.

Además, este retorno ayuda a identificar lo que hemos denominado el «ejercicio de la responsabilidad pública», que es muy pedagógico tanto para los gobernados como para los gobernantes.

Finalmente, en este ejercicio de responsabilidad pública también se deben justificar los esfuerzos y los recursos que se han invertido en el proceso deliberativo. En esta dirección, sugerimos que el mismo informe de retorno a los ciudadanos responda preguntas como "¿En qué ha mejorado el proceso participativo el Estatuto de Autonomía, la Ley de Servicios Sociales o el Plan de Prisiones de Cataluña?». Esta es la auténtica prueba del algodón para la deliberación y la participación ciudadana.

A través de esta secuencia de cinco fases, hemos presentado un conjunto ordenado de sugerencias para organizar procesos de participación y deliberación. No se trata de un protocolo, en el sentido cerrado con el que este término se asocia a menudo, sino más bien de una brújula para orientarnos.

Ahora nos hace falta experimentar, equivocarnos, aprender e ir perfeccionando la manera de favorecer estos debates que, recordémoslo, deben contribuir a mejorar las políticas y, por lo tanto, el bienestar de la gente. Este es, en nuestra opinión, el único criterio de evaluación de la participación que nos debe preocupar de verdad. 